Measurement of Transverse Single Spin

Asymmetries in $\pi^{0}$ Production from $p^{\uparrow}+p$ and $p^{\uparrow}+A$ Collisions at STAR

\title{
Christopher Dilks*
}

Pennsylvania State University

E-mail: cjd5150@psu.edu

In 2015 the first collisions between polarized protons and nuclei occurred at the Relativistic Heavy Ion Collider (RHIC), at a center-of-mass energy of $\sqrt{s_{N N}}=200 \mathrm{GeV}$. Comparisons between spin asymmetries and cross-sections in $p+p$ production to those in $p+A$ production provide insight into nuclear structure, namely nuclear modification factors, nuclear dependence of spin asymmetries, and comparison to models with saturation effects. The transverse single-spin asymmetry, $A_{N}$, has been measured in $\pi^{0}$ production in the STAR Forward Meson Spectrometer (FMS), an electromagnetic calorimeter covering a forward psuedorapidity range of $2.6<\eta<4$. Within this kinematic range, STAR has previously reported the persistence of large $\pi^{0}$ asymmetries with unexpected dependences on $p_{T}$ and event topology in $p+p$ collisions. This talk will compare these dependences to those in $p+A$ production.

XXIV International Workshop on Deep-Inelastic Scattering and Related Subjects

11-15 April, 2016

DESY Hamburg, Germany

\footnotetext{
* Speaker.
} 


\section{Introduction}

One of the principal observables which helps to gain insight on the spin and orbital angular momenta of partons within polarized protons is the transverse single-spin asymmetry, denoted $A_{N}$. Letting $d \sigma^{\uparrow(\downarrow)}$ denote the differential cross-section for leftward scattering of a spin up (down) polarized proton on an unpolarized target, $A_{N}$ is defined as the following ratio:

$$
A_{N}:=\frac{d \sigma^{\uparrow}-d \sigma^{\downarrow}}{d \sigma^{\uparrow}+d \sigma^{\downarrow}} .
$$

This asymmetry shows up as a $\cos \phi$ modulation of the cross-section, where $\phi$ is the azimuth of the observed particle. Since 1976, large values of $A_{N}$ have been seen in forward pion production, with only modest dependence on the center-of-mass energy, $\sqrt{s}$ [1]. For forward $\pi^{0} \mathrm{~s}, A_{N}$ increases with respect to $x_{F}:=2 p_{z} / \sqrt{s}$, where $p_{z}$ is the longitudinal momentum of the $\pi^{0}$, and $A_{N}$ is mostly flat or rising with respect to $\pi^{0}$ transverse momentum, $p_{T}$. [2][3][4].

In 2015, the world's first polarized $p^{\uparrow}+A$ synchrotron collisions took place at the Relativistic Heavy Ion Collider (RHIC), at $\sqrt{s}=200 \mathrm{GeV}$. Significant data samples of polarized protons colliding against gold $(A=197)$ and aluminum $(A=27)$ nuclei were obtained. With these new data, there are a few observables and possible implications available. The first natural question from a spin physics point of view is how $A_{N}$ in $p^{\uparrow}+A$ compares to that in $p^{\uparrow}+p$ collisions. One implication of this comparison is its use as a possible probe of gluon saturation: the color glass condensate model predicts that $A_{N}$ in $p^{\uparrow}+A$ decreases as $A$ increases [5]. The nuclear modification factor, $R_{p A}$, and its kinematic-dependences can also be assessed. Dependences of $A_{N}$ on event topology can also be compared between $p^{\uparrow}+A$ and $p^{\uparrow}+p$ collisions, possibly leading to insight on fragmentation universality. Finally, dependences of $A_{N}$ as well as $R_{p A}$ on collision centrality is another avenue for exploring possible effects a nuclear medium has on these observables.

\section{Forward Calorimetry at STAR}

The primary detector used in this analysis is the Forward Meson Spectrometer (FMS), a Pbglass electromagnetic calorimeter covering a pseudorapidity range of $2.6<\eta<4$. It is composed of 1,264 Pb-glass cells, each coupled to photomultiplier tubes, arranged in a square array as shown in Figure 1. The primary observable is the $\pi^{0} \rightarrow \gamma \gamma$ decay channel, where the transverse distance between the two photons along with their energies provide access to the di-photon invariant mass, $M_{\gamma \gamma}$, and subsequently to $\pi^{0}$ reconstruction.

Another detector, which is used in quantifying systematic uncertainties in this analysis, is the pair of Beam Beam Counters (BBCs). Each BBC is an annular arrangement of hexagonally-tiled scintillator panels, as shown in the left side of Figure 2. The portion of the BBC used in this analysis covers a pseudorapidity range of $3.3<|\eta|<5$, where $\eta>0$ denotes forward production (i.e., toward the FMS) and $\eta<0$ denotes backward production (i.e., opposite the FMS). In $p+A$ collisions, the proton beam faces the FMS and the nuclear beam remnants scatter toward $\eta<0$.

In order to reconstruct $\pi^{0} \mathrm{~s}$, photons within $35 \mathrm{mrad}$ isolation cones were collected. Within each event, only the highest energy photon pair in the highest energy isolation cone cluster was considered. This event selection yielded a $M_{\gamma \gamma}$ distribution with a lower background than lessrestrictive event selections. To select $\pi^{0}$ s from di-photon events, a mass cut of $\left|M_{\gamma \gamma}-135\right|<$ 


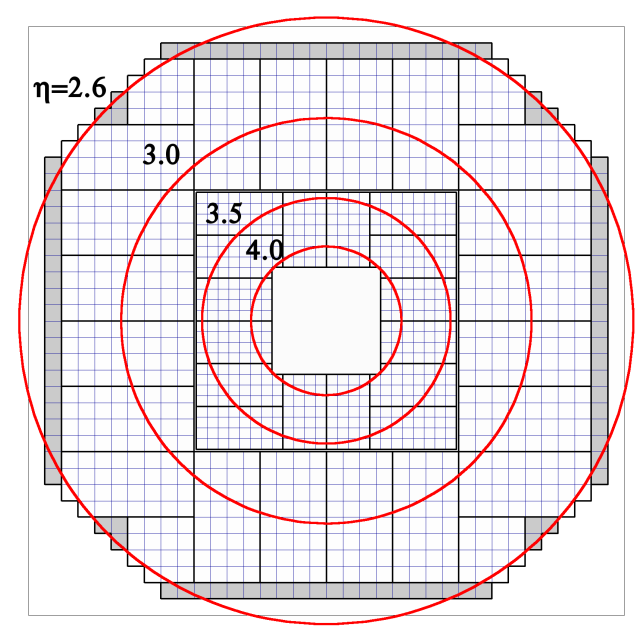

Figure 1: Schematic of the FMS Pb-glass cells. Red circles indicate rings of constant $\eta$, bold black doublelines indicate the boundary between large outer cells and small inner cells, and grey-colored cells are not in the trigger. The beam-pipe passes through the white square in the center.

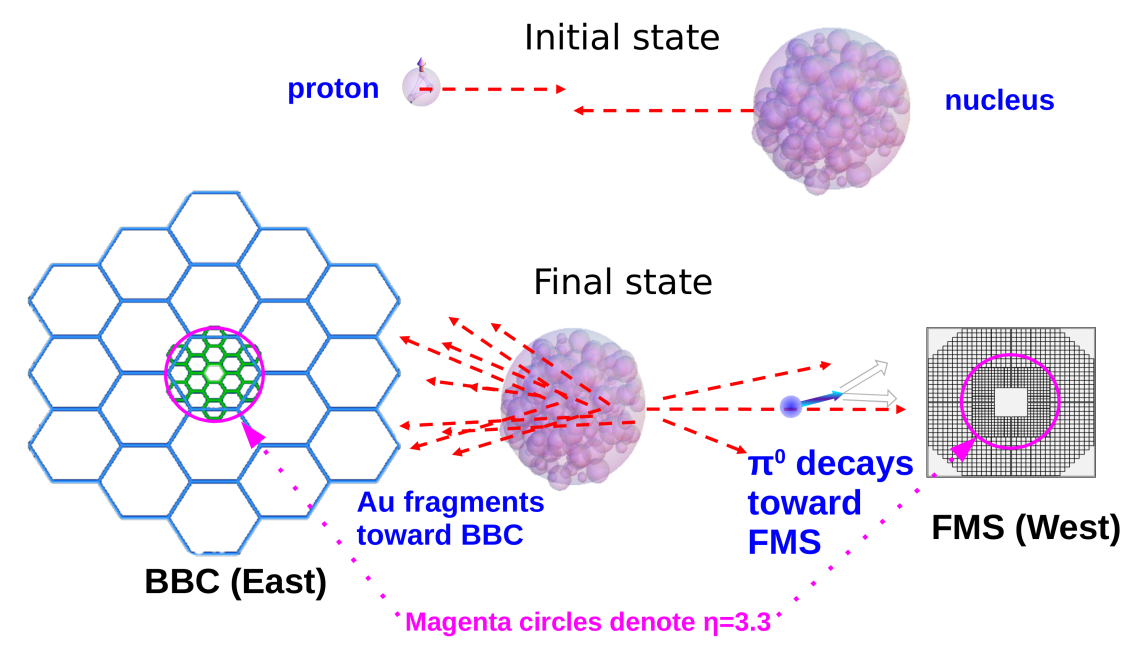

Figure 2: Schematic of the $p+A$ experimental setup, with the FMS and BBC shown in the final-state setup. The proton beam faces the FMS and the nuclear beam faces the opposite $\eta<0$ BBC. Only the scintillators inside the pink $\eta=3.3$ ring are considered in this analysis; this $\eta$-ring is also drawn on the FMS schematic for comparison.

$120 \mathrm{MeV} / c^{2}$ was used, along with an energy-sharing cut of $\left|E_{1}-E_{2}\right| /\left(E_{1}+E_{2}\right)<0.7$, where $E_{1,2}$ denote the energy of the photons. Finally, after demanding the $\pi^{0} p_{T}$ to be above trigger threshold, these $\pi^{0} \mathrm{~s}$ were then organized into $E$ and $p_{T}$ bins for subsequent asymmetry analysis.

The selected $\pi^{0} \mathrm{~s}$, within their $E$ and $p_{T}$ bins, are then binned in $\cos \phi$ bins. Letting $N^{\uparrow(\downarrow)}$ denote the $\pi^{0}$ yield from the scattering of spin-up(down) protons, the distribution of the raw trans- 
verse single-spin asymmetry is fit to the following linear equation with parameters $p_{0,1}$ :

$$
\frac{N^{\uparrow}-N^{\downarrow}}{N^{\uparrow}+N^{\downarrow}}=p_{0}+p_{1} \cos \phi
$$

The measured $A_{N}$ is then the amplitude of the azimuthal modulation of this quantity, divided by the beam polarization $P$, that is, $A_{N}=p_{1} / P$, whereas $p_{0}$ scales with the relative luminosity between spin up and spin down protons.

In $p+A$ collisions (and to a lesser extend in $p+p$ collisions), the extracted $A_{N}$ depends on the away-side BBC multiplicity, i.e., on the charged particle distribution from the nuclear remnants. In this analysis, this dependence is characterized as the dominant systematic uncertainty on $A_{N}$ within each kinematic bin; further characterization of this dependence and its possible relation to centrality will be studied in future analyses.

\section{Results and Discussion}

The transverse single-spin asymmetries for forward $\pi^{0}$ s produced in $p+p$ and in $p+A u$ collisions are shown as a function of $p_{T}$ in six different $x_{F} \approx E_{\pi^{0}} /(100 \mathrm{GeV})$ bins in Figure 3, where the $x_{F}$ approximation is valid for forward production kinematics; filled-in points are for $\pi^{0} \mathrm{~s}$ from $p+p$ and open points are those from $p+A u$. Statistical uncertainties are represented by vertical error bars and the dominant systematic uncertainty from the charged particle multiplicity in the gold-going BBC is represented by the vertical size of the grey band centered at $A_{N}=-0.005$. The asymmetry from $p+p$ is similar to that from $p+A u$, within statistical and systematic uncertainties. These data represent a luminosity of approximately $35 \mathrm{pb}^{-1}$ from $p+p$ and $205 \mathrm{nb}^{-1}$ from $p+A u$. Forward proton beam average polarizations were $55.6 \pm 2 \%$ and $60.4 \pm 2 \%$ for $p+p$ and for $p+A u$, respectively.

Another study that was performed with data from the 2015 RHIC run is the dependence of $\pi^{0}$ $A_{N}$ on event topology. Recent data from the FMS in previous RHIC runs have shown that isolated $\pi^{0}$ s have a higher $A_{N}$ than those which are surrounded by other forms of electromagnetic energy [3][4]. This dependence was studied again in the $2015 p+p$ data and was compared to the $p+A u$ sample. For a given $\pi^{0}$ in a 35 mrad isolation cone, the next highest energy photon cluster with $E>3 \mathrm{GeV}$ outside the isolation cone was considered; its $(\eta, \phi)$-position relative to that of the $\pi^{0}$ was denoted $(\Delta \eta, \Delta \phi)$. The $\pi^{0}$ events were then separated into two isolation classes: those with electromagnetic energy deposited $\Delta \phi<100 \mathrm{mrad}$ from the $\pi^{0}$ and its more-isolated complement, $\Delta \phi>100 \mathrm{mrad}$. Figure 4 shows the $A_{N}$ for the two event classes, where more-isolated $\pi^{0} s$ are plotted as filled-in squares and less-isolated as open circles. The same trend persists in $p+A u$ : more isolated $\pi^{0} s$ have a higher $A_{N}$. 

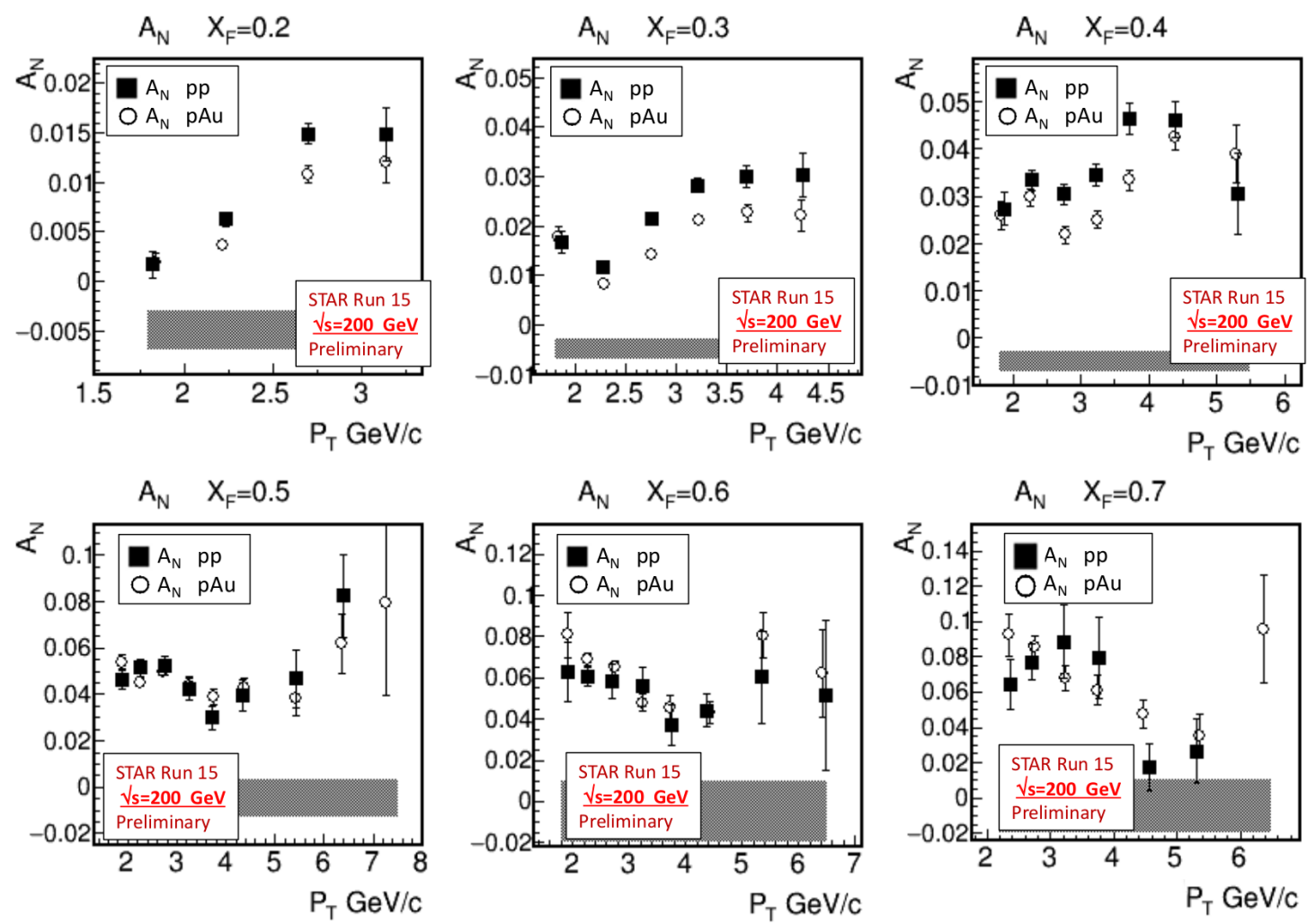

Figure 3: Transverse single-spin asymmetry $A_{N}$ vs. $p_{T}$ for $\pi^{0}$ s from $p+p$ (filled points) compared to $p+A u$ (open points) for six $x_{F}$ bins of widths 0.1 centered about the value indicated in the plot titles. Vertical error bars are statistical uncertainties and the vertical size of the grey band centered around $A_{N}=-0.005$ is the size of the systematic uncertainty from the BBC multiplicity. (Note: vertical scales are different in each plot)
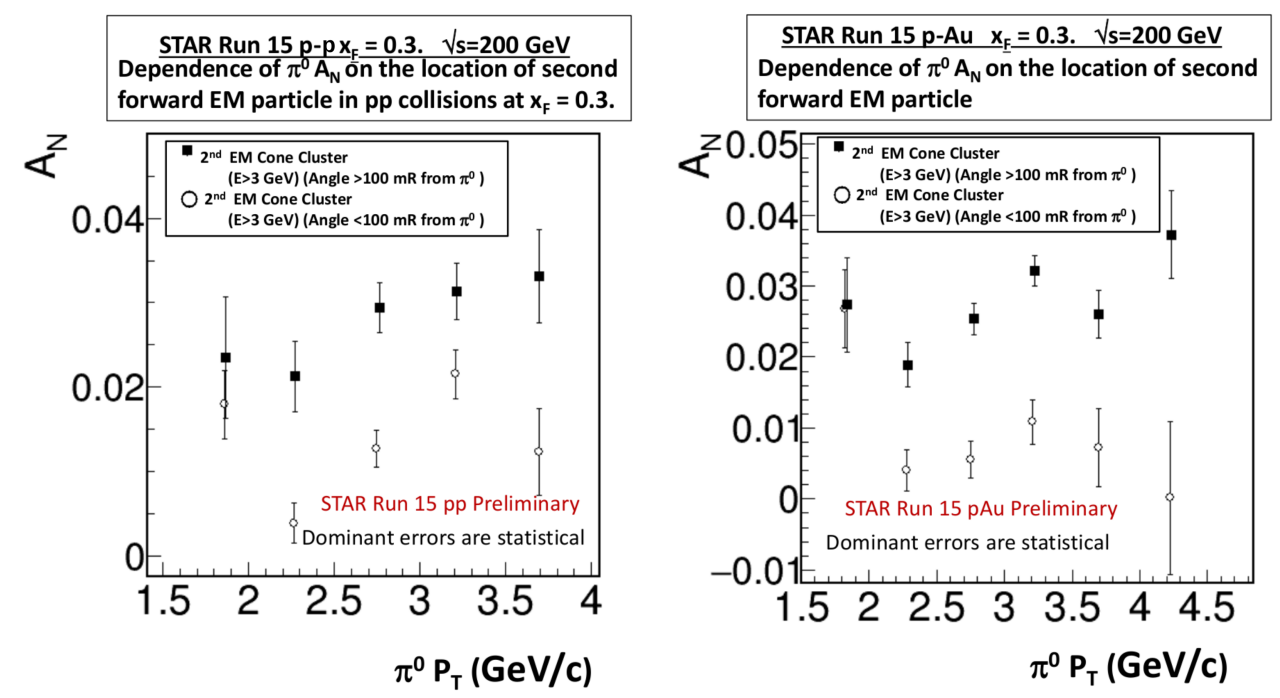

Figure 4: $A_{N}$ for isolated (filled squares) and non-isolated (open circles) $\pi^{0}$ s from $p+p$ (left) and $p+A u$ (right). (Note: vertical scales are different in each plot) 


\section{Conclusion}

This analysis was of $A_{N}$ from $\pi^{0} \mathrm{~s}$ from the world's first synchrotron collisions of polarized protons against nuclei at $\sqrt{s}=200 \mathrm{GeV}$. The value of $A_{N}$ in $p+p \rightarrow \pi^{0}+X$ was shown to be within statistical and systematic uncertainties of that in $p+A u \rightarrow \pi^{0}+X$ for all kinematic bins analyzed. The value of $A_{N}$ in both cases appears to be flat or to be rising with respect to $p_{T}$ and also rises with $x_{F}$. Its dependence on event topology is also similar for both cases: isolated $\pi^{0} \mathrm{~s}$ are associated with higher values of $A_{N}$. Plans for future analyses include characterizing more carefully the dependence of $A_{N}$ on nuclear breakup multiplicity and its possible relation to collision centrality, as well as studies of nuclear modification factors within these kinematics.

\section{References}

[1] R. D. Klem et al., Measurement of Asymmetries of Inclusive Pion Production in Proton-Proton Interactions at 6-GeV/c and 11.8-GeV/c, Phys. Rev. Lett. 36 (1976) 929.

[2] STAR Collaboration (B. I. Abelev et al.), Forward Neutral-Pion Transverse Single-Spin Asymmetries in $p+p$ Collisions at $\sqrt{s}=200$ GeV, Phys. Rev. Lett. 101 (2008) 222001 [hep-ex/08012990].

[3] S. Heppelmann, Large $p_{T}$ Forward Transverse Single Spin Asymmetries of $\pi^{0}$ Mesons at $\sqrt{s}=200$ and $500 \mathrm{GeV}$ from STAR, in proceedings of DIS 2013, POS (DIS 2013) 240 (2013).

[4] M. Mondal, Measurement of the Transverse Single-Spin Asymmetries for $\pi^{0}$ and Jet-like Events at Forward Rapidities at STAR in $p+p$ Collisions at $\sqrt{s}=500 \mathrm{GeV}$, in proceedings of DIS2014, PoS (DIS2014) 216 (2014), [nucl-ex/14073715].

[5] Z. B. Kang and F. Yuan, Single Spin Asymmetry Scaling in the Forward Rapidity Region at RHIC, Phys. Rev. D 84 (2011) 034019 [hep-ph/11061375]. 